\title{
Improved Ordinary Measure and Image Entropy Theory based intelligent Copy Detection Method*
}

\author{
Dengpan Ye ${ }^{1,2,3}$, Longfei Ma², Lina Wang ${ }^{2,3}$, Robert H. Deng ${ }^{1}$ \\ ${ }^{1}$ School of Information System, Singapore Management University, 178902, Singapore \\ ${ }^{2}$ School of Computer Science, Wuhan University, Wuhan, Hubei, 430072, China \\ ${ }^{3}$ The Key Laboratory of Aerospace Information Security and Trust Computing, Ministry of Education, \\ Wuhan, Hubei, 430072, China \\ E-mail: yedp2001@163.com
}

\begin{abstract}
Nowadays, more and more multimedia websites appear in social network. It brings some security problems, such as privacy, piracy, disclosure of sensitive contents and so on. Aiming at copyright protection, the copy detection technology of multimedia contents becomes a hot topic. In our previous work, a new computer-based copyright control system used to detect the media has been proposed. Based on this system, this paper proposes an improved media feature matching measure and an entropy based copy detection method. The Levenshtein Distance was used to enhance the matching degree when using for feature matching measure in copy detection. For entropy based copy detection, we make a fusion of the two features of entropy matrix of the entropy feature we extracted. Firstly, we extract the entropy matrix of the image and normalize it. Then, we make a fusion of the eigenvalue feature and the transfer matrix feature of the entropy matrix. The fused features will be used for image copy detection. The experiments show that compared to use these two kinds of features for image detection singly, using feature fusion matching method is apparent robustness and effectiveness. The fused feature has a high detection for copy images which have been received some attacks such as noise, compression, zoom, rotation and so on. Comparing with referred methods, the method proposed is more intelligent and can be achieved good performance.
\end{abstract}

Keywords: Ordinal Measure; Image Entropy Theory; Copy Detection.

\section{Introduction}

Nowadays, internet technology and multimedia technology develop so fast. Many of us marvel at the novelty of internet sites that seem to appear from everywhere. Accompany with the technology, a new social network platform [1] which is used for media creating, publishing and sharing has become the hot spot.

YouTube is a good example. YouTube, which founded in February 2005, is a typical of internet companies. It originated in a garage, followed quickly by significant funding from a venture capital company, in this case, Sequoia Capital. At first, users shared personal videos, but as the Web site's popularity grew rapidly, YouTube 
contracted with traditional content providers (television and movie studios and record labels) to load commercial clips. In less than a year, the video and user statistics were staggering. The company claims that 65,000 videos are uploaded daily, with consumers watching more than 100 million videos a day. Twenty million unique users, mainly in the 18-49 age range, view the Web site monthly [2]. In YouTube site, one can upload and share their videos conveniently and frequently.

Due to the media content can be download and upload freely, the freedom also brings some serious content security problems, such as some harmful content's spread, the content may include some sensitive contents related to sex, terror, privacy and pirate and so on. Therefore, the manager of the website has the responsibility to check the media contents which were uploaded to the website to make sure whether there are some sensitive contents in it or not. In the website, someone may also upload the media that others had already uploaded, in order to protect the original author's copyright and save the space of the server disk, the website manager should prevent the same media's uploading. Nowadays, the dominant method of checking the media contents is manual operation. But when the numbers of the media increasing so fast, it is obvious that the manual method cannot complete this complex task efficiently. Therefore, a new Computer-based Control System used to detect the media has been proposed in our previous work [1].

In this paper, we continue do some research on the Computer-based Control System. An improved media feature matching measure using Levenshtein Distance [3] and a new image entropy theory [4, 5, 6] based feature extraction and matching measure are proposed in this paper.

The rest of the chapter is arranged as follows. Section 2 reviews related works. The improved matching measure for ordinary measure is shown in section 3 . The entropy theory based media features extraction and matching measure is proposed in section 4 . Section 5 presents the experiments and makes a comparison between the two proposed methods and the related methods. Final conclusion is shown in section 6 .

\section{Related works}

Copy detection is an important aspect of copyright protection. Many domestic and foreign scholars had done a lot of research in this field.

In our previous work, we have proposed a computerbased copyright control system [1] and a simple grayscale-based ordinary measure of feature extraction method which is efficient for image copy detection. Paper [7] proposed a new framework called the extended feature set for detecting copies of images. Firstly, virtual prior attacks are applied to copyrighted images to generate novel features, which serve as training data. Then the copy-detection problem can be solved by learning classifiers from the training data. This approach can be integrated into existing copy detectors to further improve their performance, but low efficiency for unknown attack type. In [21], an anisotropic wavelet-Based intelligent method was used for image nearness measuring but the detection results are fuzzy not definite decision. Paper [8] used the video and audio fingerprint method to resist the attacks. Among the features, Audio copy detection appears to be more robust due to relatively simple nature of attacks. However, as seen in the experiments visual features cannot withstand some of the attacks such as picture-inpicture attack. Furthermore, the author improved the result by joint usage of visual and audio features. In next part, several related works detail will be shown.

\subsection{Computer-based Copyright Control System}

According to the emergence of numerous media in social network at present, checking all the media through manual operation is unrealistic. In paper [1], a new Computer-based Copyright Control System is proposed to deal with this complex problem. The system is focus on how to prevent the conflict of the copyright in social network.

The system is defined as Eq. (1):

$$
S=\{\text { Media Content, MFD, Media Certification }\}
$$

Media Content represents the content that the user prepares to upload. MFD represents the media feature database which stores the registered content's feature. Media Certification represents the detective process. The automatic content review mechanism is shown in Fig. (1). 


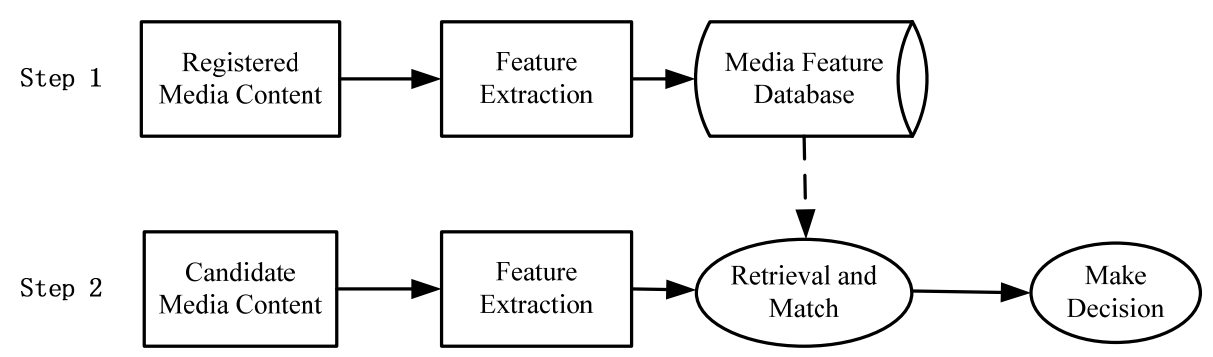

Fig. 1 The automatic content review mechanism of the System

In the system, two targets are aimed to be achieved. First, the upload content cannot conflict with the content which was already exist in the database and identified as the well-known manufacturing companies. Second, the upload content cannot conflict with the content which was already manufactured and uploaded by other users. So, in order to achieve these two targets, two steps should be done as:

Step 1: initialize the MFD with efficient media feature extraction method.

Step 2: extract the feature of the candidate media content using the same extraction as initialization, then use high-efficient measure to retrieval and match the candidate media's feature. At last, make a decision.

If match succeed, the upload content will be prevented, else the content will be permitted to upload.

\subsection{Ordinary measure based feature extraction and matching measure}

In paper [1], a grayscale ordinary measure [9, 10, 11, 12, and 13] based media feature extraction and a simple matching measure were presented.

For a image (Fig. (2)), firstly, partition the image into $\mathrm{N}$ blocks $(\mathrm{N}=\mathrm{n} \times \mathrm{n}, \mathrm{n}$ denote the number of column or row, in Fig. (3)), if the image is a kromogram, the grayscale linear transformation can be used to preprocess the picture into a gray one.

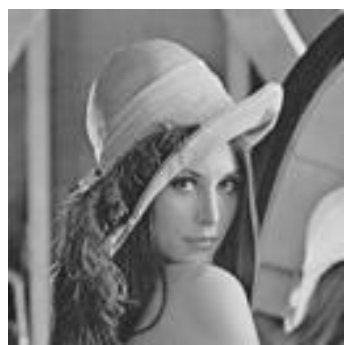

Fig. 2 Lena

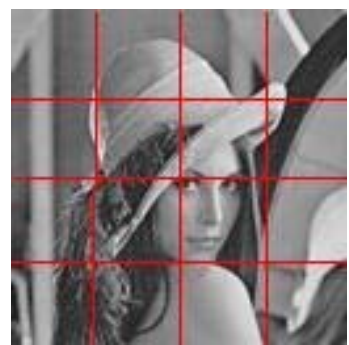

Fig. 3 Partitioned image
Secondly, calculate the average grayscale value of every block, then, a gray value matrix $M(\mathrm{mij}, \mathrm{i}, \mathrm{j}=1,2 \ldots \mathrm{n})$ can be gotten, then transform the 2-D matrix $M$ into 1-D array $\mathrm{B}(\mathrm{bk}$, ), the length of the array is $\mathrm{N}=\mathrm{n} \times \mathrm{n}$.

At last, an index sequence of the $\mathrm{S}(\mathrm{Sk}, \mathrm{k}=1,2 \ldots \mathrm{N})$ can be gotten after ascending sorting the block array by the grayscale value. As shown in Eq. (2), S1 denotes the index of the block which has the smallest grayscale value. Sk denotes the index of the block which has the kth smallest grayscale value.

$$
S=\left(S_{1}, S_{1}, \cdots S_{k}, S_{N}\right)=\operatorname{Sort}(B)
$$

In paper [1], the author used the error-rate (ER) to represent the degree of the error match. Suppose if there are two sequences, including an initial image's media feature sequence (seqA) and a test image's media feature sequence (seqB). Then calculate the differences of the two sequences as the ER with Eq. (3) and (4). The smaller the error-rate is, the bigger matching probability is, in other words, the image is more probable to be judged as a duplicated one.

$$
\begin{gathered}
\text { Equal }(\operatorname{seq} A, \operatorname{seq} B)_{i}=\begin{array}{lr}
0 & \operatorname{seq}_{i} \neq \operatorname{seq} B_{i} \\
1 & \operatorname{seq} A_{i}=\operatorname{seq} B_{i}
\end{array} \\
E R=\frac{\sum_{i=1}^{N} \text { Equal }(\operatorname{seq} A, \operatorname{seq} B)_{i}}{N}
\end{gathered}
$$

The measure can resist some attacks such as compress, zoom, gaussian noise and so on. The experiments showed that the method is robust and efficient. But this measure is low efficient when facing rotated image.

\subsection{Information entropy theory and Image entropy}

In 1948, Shannon had proposed the conception of information entropy [14]. In information theory [15], the information entropy is defined as mathematical expectation of the extraneous variable during the set $\{X$, $P(x)\}$. It can be described as the mathematical equation:

$$
H(x)=E\left[\log \frac{1}{P\left(x_{i}\right)}\right]=-\sum_{i=1}^{L} P\left(x_{i}\right) \cdot \log P\left(x_{i}\right)
$$


In the case of pi $=0$ for some $i$, the value of the corresponding summand $0 \log (0)$ is taken to be 0 , which is consistent with the limit:

$$
\lim _{p \rightarrow 0+} p \log p=0
$$

Based on the entropy theory, we can define the digital image's grayscale entropy [4] [5] [6]. In digital image, the picture consists of numerous pixels. The grayscale value of the pixels' probability distribution is different from each other, so every image has its own feature. Represent the grayscale value as the set $X$, suppose there is of a pixel which grayscale value is $m$, and the probability of the pixel's appearance in this image is $\mathrm{P}(\mathrm{m})$, so according to the definition of the information entropy theory, the image grayscale entropy can be described as :

$$
H(X)=E\left[\log \frac{1}{P(m)}\right]=-\sum_{m=0}^{255} P(m) \log P(m)
$$

Paper [4] proposed an entropy based image retrieval algorithm. The algorithm is described as follow:

(i)Remove the noise of the image.

(ii)Map the image on $n \times n$ grid, then calculate the entropy of every cell of the grid and get a entropy matrix.

$$
Q=\begin{array}{cccc}
Q_{11} & Q_{12} & \cdots & Q_{1 n} \\
Q_{21} & Q_{22} & \cdots & Q_{2 n} \\
\vdots & \vdots & \ddots & \vdots \\
Q_{n 1} & Q_{n 1} & \cdots & Q_{n n}
\end{array}
$$

(iii)Calculate matrix Q's eigenvalue, then an eigenvalue vector $\left(\lambda_{1}, \lambda_{2}, \cdots \lambda_{n}\right)$ can be gotten after sort the eigenvalue by norm, $\left(\left\|\lambda_{1}\right\| \geqslant\left\|\lambda_{1}\right\| \geqslant \ldots \geqslant\left\|\lambda_{1}\right\|\right)$. Next, pick up parts of the eigenvalue for the entropy feature according to Eq. (9). $\beta$ is a predefined threshold, $\beta \in[0,1]$.

$$
\left(\sum_{i=1}^{t} \lambda_{i}\right) /\left(\sum_{i=1}^{n} \lambda_{i}\right) \geqslant \beta
$$

(iv)Calculate the Euclidean Distance of the vector according to Eq. (10).

$$
d=\sqrt{\sum_{i=1}^{t}\left\|\lambda_{i} \quad \lambda_{i}^{\prime}\right\|^{2}}
$$

The algorithm has high efficient when the image encounter rotation or zoom. But, it is low efficient when facing a noised image or a grayvalue-changed image.

\section{Improved feature matching method for ordinary measure}

The feature matching method presented in paper [1] is coarse, and it cannot resist the local tamper image when the matching is not so precise. In this paper, we improve the matching method to achieve more efficient matching result.

\subsection{Levenshtein Distance}

Levenshtein distance (LD) [3] is a measure of the similarity between two strings, which we will refer to as the source string (s) and the target string (t). According to the ideas of the algorithm, we improve it, and make the algorithm suitable for calculate the similarity between two integer vectors. Then, the algorithm can be used to get the similarity of the grayscale value sequence of ordinary measure.

The process of improved Levenshtein Distance is follow:

Step 1: Initialization

Set $n=$ vectorLength(s), $m=$ vectorLength $(t)$, if $n=0$, return $\mathrm{m}$; if $\mathrm{m}=0$, return $\mathrm{n}$;

Set Matrix $G[(m+1) \times(n+1)]$ ( Fig. 4(a) ), Initialize the first row to $0 . . n$; Initialize the first column to $0 . . \mathrm{m}$.

Step 2: Iteration

For $\mathrm{j}=1$ to $n$

For $\mathrm{i}=1$ to $\mathrm{m}$

If $s[j]=t[i]$, set cost $=0$, else $\cos t=1$;

Set Cell $g[i, j]$ of the matrix $G$ equal to the minimum of ( Fig. 4(b) ):

- The cell immediately above plus 1: $\mathrm{g}[\mathrm{i}-1, \mathrm{j}]+1$;

- The cell immediately to the left plus 1: $\mathrm{g}[\mathrm{i}, \mathrm{j}-1]+1$;

- The cell diagonally above and to the left plus the cost: $g[i-1, j-1]+$ cost;

\section{End}

End

Step 3: Result

The distance $\mathrm{R}$ is found in cell $\mathrm{g}[\mathrm{m}+1, \mathrm{n}+1]$. 

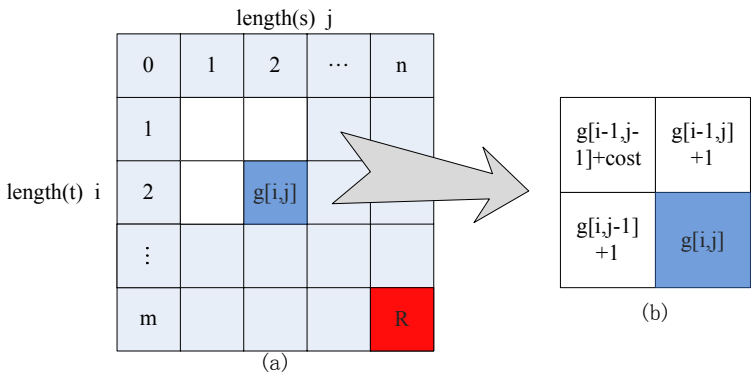

(b)

Fig. 4 Levenshtein Distance

\subsection{Feature matching based on LD algorithm}

For the grayscale ordinary measure we proposed in paper [1], after extracting the feature of the image, we store the feature (sorted grayscale sequence) in an integer vector, and then we use the LD algorithm to match the image feature, instead of the simple error-rate matching measure.

Next, we define a threshold $\lambda$ which rendered by the control system. If the matching result is lower than the threshold, we asserted that the matching is successful. In other words, the test image is the same with the original image (or tampered from the original image). Else, match failed.

$$
\text { Result }=\begin{array}{cc}
\text { Succeed } & \text { matching degree }>\lambda \\
\text { Failed } & \text { matching degree }<\lambda
\end{array}
$$

\section{Entropy based feature extraction and matching measure}

In the related works in previous section, we introduce Shannon's information entropy theory and the image entropy theory definition, and we also review an algorithm which presented in paper [4]. Here, we use an improved method based on entropy theory and matrix theory to cope with the feature extraction and matching measure respectively.
The process of the feature extraction and the matching measure is shown in Fig. (5).

\subsection{Get image feature based on information entropy}

In section 2, a simple introduction of information entropy has been presented. In this section, we will describe the image entropy based on the entropy theory particularly. For a grayscale image, it can be known that, the appearance of every pixel's grayscale value is random, and the probability of every pixel is independent of each other. With this, the probability of the different pixels' appearance can be treating as the sample of the probability distribution. So, the information entropy theory can be used in this situation, and we call it global image entropy. The definition formula of the global image entropy can be seen from Eq. (7).

According to the definition of the global image entropy, it can be known that the global image entropy can represent the image's global statistic feature and only associate with the probability of the pixel's appearance. The same image will catch the equal global image entropy value, and then the entropy can represent the image's characteristic, so the entropy can be regard as the feature of the image. But, the global image entropy has neglected the image's distribution information in spatial region, so different image may catch the same global image entropy value. Therefore, it is imperfect when considering the global image entropy alone.

- In order to solve this problem, we combine the global image entropy and the image's spatial region to deal with it. We called the image's spatial region feature as local image entropy. The process of calculating the local image entropy is following below:

Firstly, for a grayscale image, partition it to $\mathrm{N} \times \mathrm{N}$ blocks, e.g. Fig. (2) and Fig. (3). If the image is not a grayscale one, use the grayscale linear transformation to get the corresponding grayscale

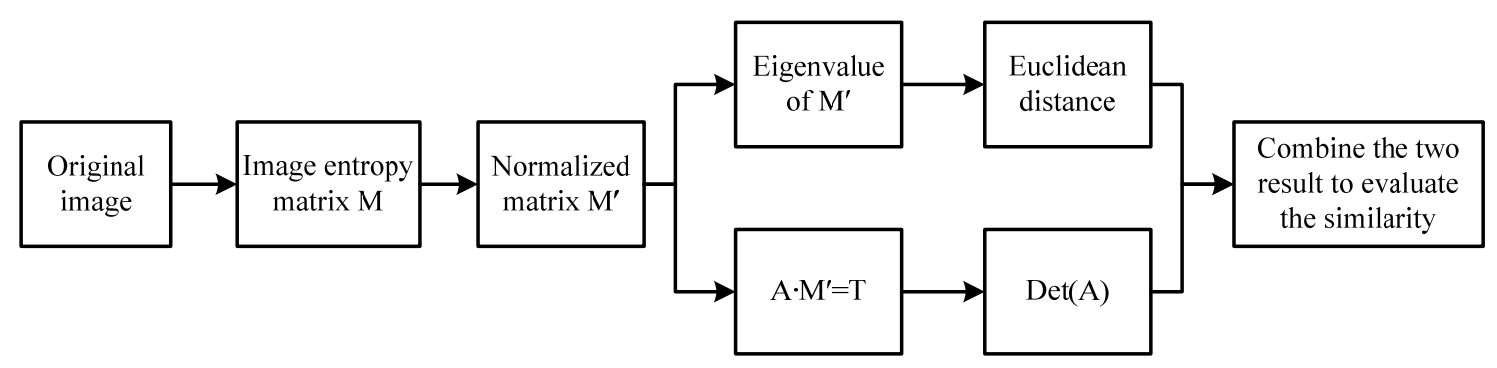

Fig. 5 Process of the feature extraction and matching measure 
image.

- Secondly, calculate every block's global image entropy with Eq. (7).

- At last, we can get an image entropy matrix $\mathrm{M}(\mathrm{N} \times \mathrm{N})$ which represents the image's global image entropy and the local image entropy. An example for the standard Lena image (Fig. 2) is shown in Table 1.

Table 1 Image entropy matrix $(\mathrm{N}=4)$

\begin{tabular}{lllll}
\hline $\mathbf{M}$ & 1 & 2 & 3 & 4 \\
\hline $\mathbf{1}$ & 6.1872 & 5.7293 & 6.6706 & 6.8044 \\
$\mathbf{2}$ & 6.0867 & 7.1461 & 6.8705 & 6.2607 \\
$\mathbf{3}$ & 6.8746 & 7.1214 & 7.0843 & 6.1211 \\
$\mathbf{4}$ & 6.8992 & 6.6555 & 6.7506 & 7.0234 \\
\hline
\end{tabular}

After getting the image entropy matrix, Gaussian normalization method can be used to transform it.

\subsection{Gaussian normalization}

Due to the dispersivity of image entropy, using the entropy value to deal with the feature directly may bring some inaccuracy. So we use the Gaussian normalization [16] [17] to normalize the image entropy value matrix. In probability theory, if an extraneous variable $\mathrm{X}$ subject to a Gaussian distribution with mathematical expectation $\mu$ and standard deviation $\sigma$, then sign it as $X \sim N\left(\mu, \sigma^{2}\right)$. The distribution can be transformed to $Z=(x-\mu) / \sigma \sim N(0,1)$.

According to " $3 \sigma$ " rule [18], if Z subject to standard normal distribution, the probability of normal variable falling in the region $(\mu-3 \sigma, \mu+3 \sigma)$ is $99.74 \%$, so the transformation: $Z=Z / 3$ will almost restrict $Z \in(-1,1)$. Furthermore, the transformation $Z=(Z+1) / 2$ will make sure that $Z \in(0,1)$.

Through the above analysis, we can get the Gaussian normalization as following:

$$
Z=\left(\frac{X-\mu}{3 \cdot \sigma}+1\right) / 2
$$

Meanwhile, there are $1-99.74 \%=0.26 \%$ of the data may be not fall in $(0,1)$, so we can treat them as following:

$$
z= \begin{cases}0, & x<0 \\ 1, & x>1\end{cases}
$$

Now, all of the data will be restrict to $(0,1)$ and achieve the normal target.
In our experiment, we treat the matrix $M$ as an array. Table 2 shows the normalized image entropy matrix $\left(M^{\prime}\right)$ of Table 1.

Table 2 Gaussian normalized image entropy matrix of Table 1

\begin{tabular}{lllll}
\hline $\mathbf{M}$ & 1 & 2 & 3 & 4 \\
\hline $\mathbf{1}$ & 0.3185 & 0.1362 & 0.5110 & 0.5644 \\
$\mathbf{2}$ & 0.2785 & 0.7004 & 0.5907 & 0.3478 \\
$\mathbf{3}$ & 0.5923 & 0.6906 & 0.6758 & 0.2922 \\
$\mathbf{4}$ & 0.6021 & 0.5050 & 0.5429 & 0.6516 \\
\hline
\end{tabular}

\subsection{Get Euclidean Distance of the matrix eigenvalue}

In mathematics, the Euclidean Distance is the "ordinary" distance between two points that one would measure with a ruler, and is given by the Pythagorean formula [19]. Euclidean Distance can measure the similarity of two vectors. The smaller the distance, the more similar the two vectors are.

The Euclidean Distance formula is shown as follow:

$$
d(X, Y)=\sqrt{\sum_{i=1}^{n}\left(X_{i} \quad Y_{i}\right)^{2}},\left(X_{i}, Y_{i}, i=1,2 \cdots n\right)
$$

In the fore step, we get the normalized image entropy matrix. Then the eigenvalues of the matrix can be calculated. Next we obtain the descending sort order of the eigenvalues by their value. If the eigenvalue is a complex number, use its module value instead. Then an eigenvalue vector (not eigenvector) can be found as $\lambda_{1}, \lambda_{2}, \cdots, \lambda_{n}$.

When matching the feature is needed, we always calculate the test image's eigenvalue vector and sort the eigenvalue elements by their value. We set the sorted vector as $\lambda_{1}{ }^{\prime}, \lambda_{2}{ }^{\prime}, \cdots, \lambda_{n}{ }^{\prime}$. At last, we use the Eq. (15) to calculate the distance of the two vectors. We can get:

$$
\operatorname{Dist}\left(M^{\prime}, T\right)=\sqrt{\sum_{i=1}^{n}\left(\lambda_{i}-\lambda_{i}^{\prime}\right)^{2}}
$$

\subsection{Calculate the determinant value of the transfer matrix}

For a test image, we can always use the same way as mentioned above to get the image entropy matrix and normalized it. Then we name the matrix $\mathrm{T}$.

So we can calculate the transfer matrix which can transfer the original image's entropy matrix $M^{\prime}$ to matrix $\mathrm{Y}$. The transfer formula is shown as follow:

$$
A \cdot M^{\prime}=T
$$


Then, we can use the matrix inverse transformation to get transfer matrix A (Eq. (17)).

$$
A=T \cdot M^{1^{-1}}
$$

According to the matrix theory, we know that when the matrix $M^{\prime}$ is similar with matrix $\mathrm{Y}$, the corresponding determinant value of $\mathrm{A}$ ( $\mathrm{A}$ is a square matrix) is more approximate to integer 1 . Therefore, we can use the suitable method to evaluate the similarity of the two matrixes.

One of the evaluate function can be seen as follow:

$$
\operatorname{Similarity}\left(M^{\prime}, T\right)=\sqrt{[\operatorname{det}(A)-1]^{2}}
$$

\subsection{Combine the two result of above methods to evaluate}

In some particular situation, one of the above methods cannot evaluate the similarity correctly, so we combine the two evaluate methods.

Lots of experiments show that the Euclidean Distance value of the eigenvalue vector is more important than the determinant value of the transfer matrix to distinguish that the test image is a duplicated (or tampered) one or not. So a suitable mathematic function to deal with the combination is expected to be found. A common one can be seen from Eq. (19).

$$
R=\operatorname{Dist}\left(M^{\prime}, T\right) \times \sqrt{\operatorname{Similarity}\left(M^{\prime}, T\right)}
$$

In Eq. (19), the efficiency of Similarity $\left(M^{\prime}, T\right)$ in combination is reduced. The final result $\mathrm{R}$ represents the evaluation of the matching result. And we can predefine a threshold $\beta$ which is obtained from numerous experiments. If $R>\beta$, we think that the two images are not similar to each other, in other words, the two images are unmatched, else if $R<\beta$, we think that the two images are matched.

\section{Experiment and analysis}

\subsection{Optimal partition}

When partitioning the image into $\mathrm{N} \times \mathrm{N}$ blocks, in theory, the bigger $\mathrm{N}$, the more efficiency the matching is. But from the result of experiment, we find it is not so. Besides, the bigger $\mathrm{N}$ will bring large amount of computation. Considering all the factors, it is important to find out the optimal partition number $\mathrm{N}$ to partition the image.

During the previous research in paper [1], we have tested the image's media feature sequence with different counts of blocks in original image and the duplicate image which is tampered with the Gaussian noise. The experiment result shows that the optimal partition number is 4 when testing the images with $128 \times 128$ size.

\subsection{Matching experiment with Levenshtein Distance (LD)}

According to the optimal partition, we partition our experimental images which sizes are $128 \times 128$ pixels into $4 \times 4$ blocks.

To evaluate our matching measure with Levenshtein Distance, several kinds of images are tested. One kind of image is the original images which are used to contrast with the others tampered images' features. Other kinds are tampered images. The species are following:

\section{Reference images:} Original images

\section{Tempered:}

(i). Gaussian white noised images

(ii). Poisson noised images

(iii). Compressed images with $75 \%$

(iv). Scaled images with coefficient 0.8

(v). Scaled images with coefficient 1.2

(vi). Rotated images with 90 degree

(vii). Rotated images with 180 degree

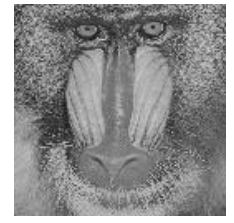

(a)

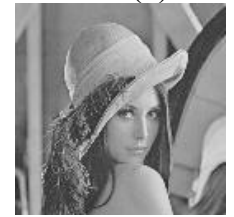

(d)

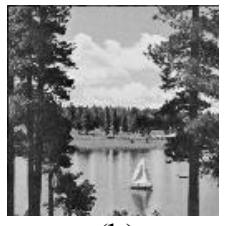

(b)

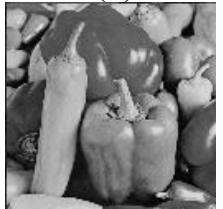

(e)

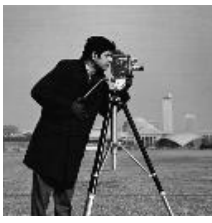

(c)

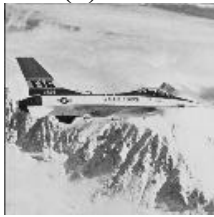

(f)
Fig. 6 Process of the feature extraction and matching measure

First, the six original test images (Fig. (6)) will be tampered by using the given temper method in front of this section. Then we calculate the grayscale value of every image, and get the index sequence of the grayscale value according to the method introduced in section 2.2. At last, we calculate the Levenshtein 
Distance of the index sequences between original images and tampered images.

After we get the Levenshtein Distance, we define the Match-Degree (MD) as Eq. (20). Levenshtein Distance measure the difference of the sequence based on quantity, when the Match-Degree represents the matching degree of the matching result.

$$
M D=1 \quad \frac{\text { Levenshtein Distance/2 }}{\text { Length of sequence }}
$$

\subsection{Comparison between LD based matching measure and simple matching measure}

italic font, we can find that the Match-Degree based on "LD" is bigger than the corresponding one based on simple matching measure. So we can know that the "LD" based matching measure is more efficient than the simple matching measure.

Owing to the little quantity of the test images, the result may be not so precise. Next, we used large amount of images to do the experiment to verify the conclusion mentioned above. In this experiment, we used 100 images (we download the images from [20]) for each kind of the tamper type and we analyze the result by statistic with the total 700 images. According to the Eq. (11), we set the threshold $\lambda=0.75$. The result of the

Table 3 Comparison of the Match-Degree between two measures

\begin{tabular}{llllllll}
\hline & & (a) & (b) & (c) & (d) & (e) & (f) \\
\hline \multirow{2}{*}{ Gauss-noise } & paper [1] & 0.9375 & 1.0000 & 0.9375 & 0.8750 & 1.0000 & 0.9375 \\
& LD & 0.9375 & 1.0000 & 0.9375 & 0.8750 & 1.0000 & 0.9375 \\
\hline \multirow{2}{*}{ Poisson-noise } & paper [1] & 1.0000 & 1.0000 & 1.0000 & 0.9063 & 0.9063 & 0.9375 \\
& LD & 1.0000 & 1.0000 & 1.0000 & 0.9375 & 0.9375 & 0.9375 \\
\hline \multirow{2}{*}{ Compress } & paper [1] & 1.0000 & 1.0000 & 1.0000 & 1.0000 & 1.0000 & 1.0000 \\
& LD & 1.0000 & 1.0000 & 1.0000 & 1.0000 & 1.0000 & 1.0000 \\
\hline \multirow{2}{*}{ Scale-0.8 } & paper [1] & 0.8750 & 0.8125 & 0.8125 & 0.7500 & 0.7500 & 0.7813 \\
& LD & 0.8750 & 0.8125 & 0.8750 & 0.7813 & 0.8125 & 0.7813 \\
\hline \multirow{2}{*}{ Scale-1.2 } & paper [1] & 1.0000 & 0.9375 & 1.0000 & 0.7500 & 0.8438 & 0.9375 \\
& LD & 1.0000 & 0.9375 & 1.0000 & 0.7813 & 0.9063 & 0.9375 \\
\multirow{2}{*}{ Rot-90 } & paper [1] & 0.5000 & 0.5000 & 0.5000 & 0.5000 & 0.5000 & 0.5000 \\
& LD & 0.5625 & 0.5000 & 0.5625 & 0.5000 & 0.5313 & 0.5625 \\
\multirow{2}{*}{ Rot-180 } & paper [1] & 0.5000 & 0.5000 & 0.5000 & 0.5000 & 0.5000 & 0.5000 \\
& LD & 0.5000 & 0.5625 & 0.5000 & 0.5313 & 0.5313 & 0.5000 \\
\hline
\end{tabular}

After obtaining the MDs between the original images and serious kinds of the tampered images through LD based matching measure, we compare it with the MDs result based on the simple matching measure which proposed in paper [1]. Table 3 shows the comparison of the two above MDs.

Just focusing on each row of "LD" from the data in Table 3, we can see that the grayscale based ordinary measure and the Levenshtein Distance based matching method is more robust when the image facing the attacks such as Gauss-noise, Poisson-noise, Compress, Scale. But we can also see from the table that the measure catch low efficiency when image is tampered with Rotation.

Next, we make a comparison between the simple matching measure (Eq. (3), (4)) which proposed in paper [1] and the LD based matching measure mentioned in section 3. Each row of "paper [1]" from the data in Table 3 shows the Match-Degree of the experiment when using the simple matching measure. Seeing from the cells which words was set by bold and experiment is shown in Table 4.

Table 4 Statistic result of large amount of images

\begin{tabular}{lccc}
\hline & $\begin{array}{c}\text { Total } \\
\text { images }\end{array}$ & paper [1] & LD \\
\hline Gauss-noise & 100 & $92 \%$ & $100 \%$ \\
Poisson-noise & 100 & $95 \%$ & $100 \%$ \\
Compress & 100 & $99 \%$ & $100 \%$ \\
Scale-0.8 & 100 & $88 \%$ & $94 \%$ \\
Scale-1.2 & 100 & $82 \%$ & $97 \%$ \\
Rot-90 & 100 & $0 \%$ & $0 \%$ \\
Rot-180 & 100 & $0 \%$ & $0 \%$ \\
\hline
\end{tabular}

From Table 4, we can see that the matching percent based on "LD" are greater than the method based on simple matching measure. Now we can declare that the "LD" based matching measure is more efficient than the simple matching measure. 
But, the grayscale ordinary measure is lower efficiency when facing the rotated tampered images. Next, we will show the image entropy based feature extraction and matching measure which is high efficient for rotation, and supply a comparison with the ordinary measure.

\subsection{Image entropy based feature extraction and matching measure}

Firstly, we do the particular experiments on the special test images, and then we use large amount of images to verify the efficiency of the method.

For images in Fig. (6), we transform the images into seven kinds of tempered images, then partition them into $\mathrm{N} \times \mathrm{N}$ blocks, and calculate the grayscale based image entropy of every block to get the entropy matrix. At last, we calculate the Euclidean Distance of the sorted eigenvalue vector of the Gaussian normalized entropy matrix using Eq. (15). The result of the transfer matrix. Then we can get the final result of the match which shown in Table 7 . In Table 7 , row 1-7, which indicate the successful match, represent the final combine result of the transfer matrix between original images and the corresponding tampered images. The last "Other image" row, which indicates the failed match, represents the final combine result between other image and the six images in Fig. (6).

Then the determinant value of the transfer matrix will be calculated in next step according to Eq. $(16,17)$. The result of the similarity value of Eq. (18) is shown in Table 6. Row 1-7 in Table 6 represent the similarity value of the transfer matrix between original images and the corresponding tampered images, and it also means the successful match. The last "Other image" row represents the similarity value of the transfer matrix between other image and the six images in Fig. (6), and it also means the failed match.

Theoretically speaking, the smaller Euclidean Distance

Table 5 Euclidean Distance of the normalized entropy matrixes' sorted eigenvalue vector

\begin{tabular}{lllllll}
\hline & $(\mathrm{a})$ & $(\mathrm{b})$ & $(\mathrm{c})$ & $(\mathrm{d})$ & $(\mathrm{e})$ & $(\mathrm{f})$ \\
\hline Gauss-noise & 0.0610 & 0.6584 & 0.1374 & 0.1560 & 0.1446 & 0.0506 \\
Poisson-noise & 0.0125 & 0.0822 & 0.0547 & 0.0263 & 0.0196 & 0.0429 \\
Compress & 0.0000 & 0.0006 & 0.0007 & 0.0000 & 0.0014 & 0.0000 \\
Scale-0.8 & 0.0391 & 0.1493 & 0.0154 & 0.0679 & 0.1390 & 0.0230 \\
Scale-1.2 & 0.0133 & 0.0775 & 0.0057 & 0.0193 & 0.0129 & 0.0078 \\
Rot-90 & 0.4301 & 0.2612 & 0.3286 & 0.2483 & 0.1719 & 0.4314 \\
Rot-180 & 0.0004 & 0.0000 & 0.0001 & 0.0001 & 0.0000 & 0.0000 \\
Other image & 0.3320 & 0.3143 & 0.3571 & 0.3953 & 0.1645 & 0.4150 \\
\hline
\end{tabular}

Euclidean Distance is shown in Table 5.

In Table 5, row 1-7 (except the last row) represents the Euclidean Distance between original images and the corresponding tampered images, in other words, it represent the successful match, and this two images are copy images. The last "Other image" row represents the Euclidean Distance between the six images (Fig. (6)) and other image which had no relation with the six images, in other words, it represent the failed match. At last, according to Eq. (19), we combine the Euclidean Distance and the determinant value of (or Similarity value) is, the more successful matching is. But in actual experiments, focusing on the Table 5 or 6 separately, it is difficult to find out a threshold which can efficiently distinguish the successful match and the failed match.

However, we combine the Euclidean Distance feature and Similarity value feature of the transfer matrix using the Eq. (19) From Table 7, we can easily find out the critical value of the successful and failed match clearly. From the experiment in Table 7, the threshold can be set as 0.25 .

Table 6 Similarity value of the transfer matrix

\begin{tabular}{lllllll}
\hline & $(\mathrm{a})$ & $(\mathrm{b})$ & $(\mathrm{c})$ & $(\mathrm{d})$ & $(\mathrm{e})$ & (f) \\
\hline Gauss-noise & 0.7494 & 0.2830 & 4.0692 & 0.3718 & 0.0700 & 1.2676 \\
Poisson-noise & 0.3719 & 0.2996 & 2.2576 & 0.0768 & 0.1275 & 1.0862 \\
Compress & 0.0000 & 0.0018 & 0.0045 & 0.0000 & 0.0053 & 0.0000 \\
Scale-0.8 & 0.2216 & 0.5844 & 0.3452 & 0.1761 & 1.3040 & 0.5357 \\
Scale-1.2 & 0.0413 & 0.1138 & 0.2726 & 0.1063 & 0.4665 & 0.0478 \\
Rot-90 & 0.1242 & 0.0245 & 0.0045 & 0.0035 & 0.1065 & 0.0624 \\
Rot-180 & 0.0058 & 0.0000 & 0.0008 & 0.0001 & 0.0000 & 0.0001 \\
Other image & 0.4271 & 4.5862 & 1.6878 & 8.5596 & 0.0473 & 0.1282 \\
\hline
\end{tabular}

Published by Atlantis Press Copyright: the authors 
Table 7 Final result of the match

\begin{tabular}{lllllll}
\hline & $(\mathrm{a})$ & $(\mathrm{b})$ & $(\mathrm{c})$ & $(\mathrm{d})$ & $(\mathrm{e})$ & $(\mathrm{f})$ \\
\hline Gauss-noise & 0.0528 & 0.3503 & 0.2772 & 0.0951 & 0.0383 & 0.0570 \\
\hline Poisson-noise & 0.0076 & 0.0450 & 0.0821 & 0.0073 & 0.0070 & 0.0447 \\
Compress & 0 & 0.0000 & 0.0000 & 0 & 0.0001 & 0 \\
Scale-0.8 & 0.0184 & 0.1141 & 0.0091 & 0.0285 & 0.1587 & 0.0168 \\
Scale-1.2 & 0.0027 & 0.0262 & 0.0030 & 0.0063 & 0.0088 & 0.0017 \\
Rot-90 & 0.1516 & 0.0409 & 0.0222 & 0.0147 & 0.0561 & 0.1078 \\
Rot-180 & 0.0000 & 0.0000 & 0.0000 & 0.0000 & 0.0000 & 0.0000 \\
\hline Other image & 0.2170 & 0.6731 & 0.4639 & 1.1566 & 0.0358 & 0.1486 \\
\hline
\end{tabular}

In order to see the distinctive degrees of the Euclidean Distance, determinant value of transfer matrix and the final combined result. We test another 18 images and get the data using the same way as the measure mentioned above. Then we plot the all of the data in three figures as Fig. (7), (8), (9). The red points represent the successful match, and the blue star points represent the failed match.

Fig. (7)represents the experiment result of the copy detection singly using Euclidean Distance feature of the entropy matrix of test images. Fig. (8) is the result of singly using determinant value feature of the transfer matrix. At last, the combined result of using both the feature in Fig. (7) and (8) is shown in Fig. (9).

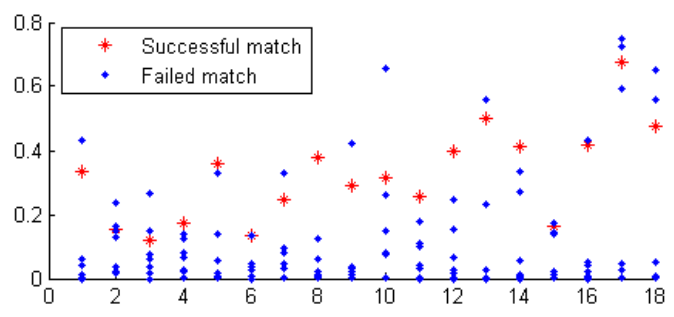

Fig. 7 Plots of Euclidean Distance data

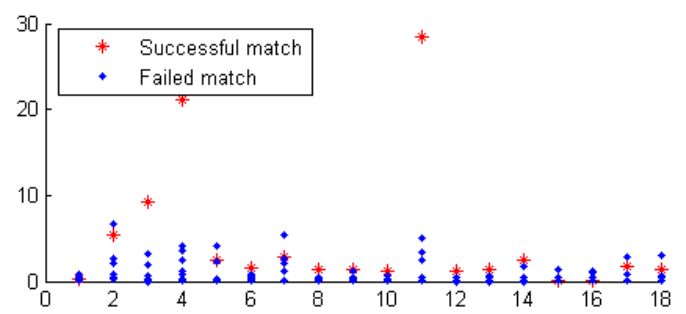

Fig. 8 Plots of Similarity of the transfer matrix

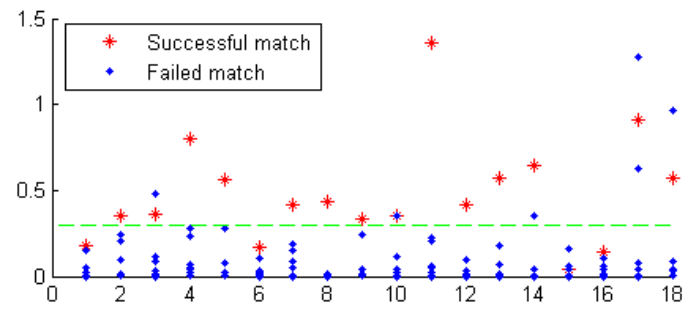

Fig. 9 Plots of combined result

It can be seen form that it is difficult to find out the threshold to divide the red points (Successful match) and blue points (Failed match) in Fig. (7) (Euclidean Distance) and Fig. (8) (The determinant value). But from the combined result in Fig. (9), we can easily find out the threshold (the green dashed line) that can divide the red points and the blue points distinctly.

\subsection{Comparison between image entropy measure and grayscale based ordinary measure}

Owing to the little quantity of the test images, the result may be not so precise. So we used large amount of images to do the experiment to verify the conclusion mentioned above, and then compare the result with the ordinary measure (paper [1]) and the measure proposed in paper [4].

Table 8 Matching results of the three measures

\begin{tabular}{lllc}
\hline & $\begin{array}{l}\text { Image } \\
\text { entropy } \\
\text { measure }\end{array}$ & $\begin{array}{l}\text { Ordinary } \\
\text { measure }\end{array}$ & $\begin{array}{c}\text { The } \\
\text { measure in } \\
\text { paper[4] }\end{array}$ \\
\hline $\begin{array}{l}\text { Gauss- } \\
\text { noise }\end{array}$ & $66 \%$ & $100 \%$ & $10 \%$ \\
$\begin{array}{l}\text { Poisson- } \\
\text { noise }\end{array}$ & $79 \%$ & $100 \%$ & $9 \%$ \\
$\begin{array}{l}\text { Compress } \\
\text { Scale-0.8 }\end{array}$ & $88 \%$ & $100 \%$ & $21 \%$ \\
Scale-1.2 & $90 \%$ & $94 \%$ & $92 \%$ \\
Rot-90 & $89 \%$ & $97 \%$ & $93 \%$ \\
Rot-180 & $92 \%$ & $0 \%$ & $98 \%$ \\
\hline
\end{tabular}


From Table 8, we can see some advantages of the image entropy measure. Comparing with the ordinary measure, the image entropy measure has high efficiency when facing the rotated images. Comparing with the measure in paper [4], the image entropy measure can detect the noised images. But it also has some weak point that the efficiency of image entropy measure is lower than the ordinary measure when images were attacked by noise, compress and scale.

\section{Conclusion}

This paper reviews the computer-based copyright control system and the media feature extraction and matching measure. Based on this system and the previous works, this paper presents an improved grayscale based ordinary measure and a new image entropy theory based feature extraction and matching measure. And we do experiments on this two proposed measure. At last, according to the experiments result, we compare the two measures and other related methods. The experiments show that both the two measures have their own advantages. The first improved ordinary measure are robust and high efficient when detecting the noised, compress, and scale images. The second image entropy theory based measure are efficient with the images which attacked by noise, compress and rotation. But the efficiency of the second measure is lower than the ordinary measure, so in the future works, some useful method such as Neural Network or other machine learning method can be used to search for the optimal threshold to increase the successful matching rate.

\section{Acknowledgements}

This work was funded by A*STAR SERC Grant of Singapore (1021010027), Natural Science Foundation of China (60903197), the foundation of the Key Laboratory of Aerospace Information Security and Trust Computing, Ministry of Education (2010) and the Fundamental Research Funds for the Central Universities.

\section{References}

1. Longfei Ma, Dengpan Ye, Shiguo Lian, Lina Wang. Computer-based copyright control system in social network and an ordinal measure based on gray scale value. 2009 , pp. $98-102$

2. http://www.infotoday.com/Searcher/may07/Ardito.shtm.

3. http://www.merriampark.com/ld.htm.

4. Sun Junding, Wu Xiaosheng, Zhou Lihua. Entropy-based image retrieval. Journal of XIDIAN University. 2004.

5. Sun Jundin, Cui Jiangtao, Liu Weiguan, Zhou Lihua. Spatial feature extraction and image retrieval based on entropy. Systems Engineering and Electronics. 2006, Vol. 01 .

6. Tian Jinwen, Yang Lei, Su Kang, Liu Jian. Image matching based on local entropy different-algorithm and computer simulation. Journal of astronautics. 1999, Vol. 1.

7. Jen-Hao Hsiao, Chu-Song Chen, Lee-Feng Chien and Ming-Syan Chen. A new approach to image copy detection based on extended feature sets. IEEE Transactions on Image Processing. 2007, Vol. 16, pp. 2069 - 2079.

8. Ahmet Saracoğlu, Ersin Esen, Tuğrul K. Ateş. Content based copy detection with coarse Audio-Visual fingerprints. 2009 Seventh International Workshop on Content-Based Multimedia Indexing. 2009, pp. 213-218.

9. Nayar, D. Bhat and S. Ordinal measures for image correspondence. IEEE Transactions on Pattern Analysis and Machine ntelligence. 1998, Vol. 20(4), pp. 415-423.

10. Hampapur, A., Bolle, R. Comparison of sequence matching techniques for video copy detection. In Conference on Storage and Retrieval for Media Databases. 2002, pp. 194-201.

11. Chen, L., Stentiford, F. W. M. Video sequence matching based on temporal ordinal measurement. s.l.: UCL Adastral, 2006.

12. Hua, X.-S., Chen, X., Zhang, H.-J. Robust video signature based on ordinal measure. In International Conference on Image Processing. 2004, Vol. 1, pp. $685-$ 688.

13. Iwamoto, K., Kasutani, E., Hamada. Image signature robust to caption superimposition for video sequence identification. In 2006 International Conference on Image Processing. 2006, pp. 3185-3188.

14. http://en.wikipedia.org/wiki/Entropy_(information_theor y).

15. http://en.wikipedia.org/wiki/Information_theory.

16. R. Jin, L. Si. A study of methods for normalizing user ratings in collaborative filtering. Proceedings of the 27 th annual international ACM SIGIR conference on Research and development in information retrieval.

17. Hofmann, T. Latent semantic models for collaborative filtering. In the Proc. of the 26th Annual International ACM SIGIR Conference. 2003.

18. http://en.wikipedia.org/wiki/Normal_distribution.

19. http://en.wikipedia.org/wiki/Pythagorean_theorem.

20. http://philip.greenspun.com/images

21. J. F. Peters and L. Puzio, "Anisotropic wavelet-based image nearness measure," International Journal of Computational Intelligence Systems, vol. 2, no. 3, pp. 168-183, 2009. 